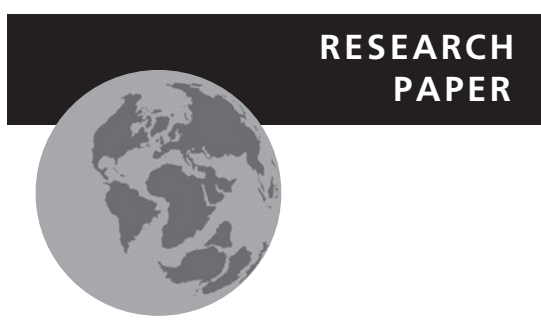

\title{
Are lizards feeling the heat? A tale of ecology and evolution under two temperatures
}

\author{
Shai Meiri ${ }^{1 \star}$, Aaron M. Bauer ${ }^{2}$, Laurent Chirio ${ }^{3}$, Guarino R. Colli ${ }^{4}$, \\ Indraneil Das ${ }^{5}$, Tiffany M. Doan ${ }^{6}$, Anat Feldman ${ }^{1}$, \\ Fernando-Castro Herrera ${ }^{7}$, Maria Novosolov ${ }^{1}$, Panayiotis Pafilis ${ }^{8}$, \\ Daniel Pincheira-Donoso ${ }^{9}$, Gary Powney ${ }^{10,11}$, Omar Torres-Carvajal ${ }^{12}$, \\ Peter Uetz ${ }^{13}$ and Raoul Van Damme ${ }^{14}$
}

${ }^{1}$ Department of Zoology, Tel Aviv University, 69978, Tel Aviv, Israel, ${ }^{2}$ Department of Biology, Villanova University, 800 Lancaster Avenue, Villanova, PA 19085, USA, ${ }^{3}$ Département de Systématique et Evolution, Muséum National d'Histoire Naturelle, 25 Rue Cuvier, 75231 Paris, France, ${ }^{4}$ Departamento de Zoologia, Universidade de Brasilia, 70910-900 Brasília, DF, Brazil, ${ }^{5}$ Institute of Biodiversity and Environmental Conservation, Universiti Malaysia Sarawak, 94300, Kota Samarahan, Sarawak, Malaysia, ${ }^{6}$ Department of Biology, Central Connecticut State University, New Britain, CT, USA, ${ }^{7}$ Departamento de Biología Facultad de Ciencias Naturales y Exactas, Universidad del Valle, Cali, Colombia, ${ }^{8}$ School of Biology, Department of Zoology and Marine Biology, University of Athens, 157-84, Panepistimioupolis, Ilissia, Greece, ${ }^{9}$ Laboratory of Evolutionary Ecology of Adaptations, School of Life Sciences, University of Lincoln, Riseholme Campus, Lincoln, Lincolnshire LN2 2LG, UK, ${ }^{10}$ NERC Centre for Ecology and Hydrology, Maclean Building, Benson Lane, Crowmarsh Gifford, Wallingford, Oxfordshire OX10 8BB, UK, ${ }^{11}$ Department of Life Sciences, Imperial College London, Silwood Park, Ascot SL5 7PY, UK, ${ }^{12}$ Escuela de Biología, Pontificia Universidad Católica del Ecuador. Avenida 12 de Octubre y Roca, Apartado 17-01-2184, Quito, Ecuador, ${ }^{13}$ Center for the Study of Biological Complexity, Virginia Commonwealth University, Richmond, VA 23284, USA, ${ }^{14}$ Department of Biology, University of Antwerp, Universiteitsplein 1, B-2610, Wilrijk, Belgium

${ }^{\star}$ Correspondence: Shai Meiri, Department of Zoology, Tel Aviv University, Tel Aviv, 69978, Israel.

E-mail: uncshai@post.tau.ac.il

\section{ABSTRACT}

Aim Temperature influences most components of animal ecology and life history - but what kind of temperature? Physiologists usually examine the influence of body temperatures, while biogeographers and macroecologists tend to focus on environmental temperatures. We aim to examine the relationship between these two measures, to determine the factors that affect lizard body temperatures and to test the effect of both temperature measures on lizard life history.

Location World-wide.

Methods We used a large (861 species) global dataset of lizard body temperatures, and the mean annual temperatures across their geographic ranges to examine the relationships between body and mean annual temperatures. We then examined factors influencing body temperatures, and tested for the influence of both on ecological and life-history traits while accounting for the influence of shared ancestry.

Results Body temperatures and mean annual temperatures are uncorrelated. However, accounting for activity time (nocturnal species have low body temperatures), use of space (fossorial and semi-aquatic species are 'colder'), insularity (mainland species are 'hotter') and phylogeny, the two temperatures are positively correlated. High body temperatures are only associated with larger hatchlings and increased rates of biomass production. Annual temperatures are positively correlated with clutch frequency and annual longevity, and negatively correlated with clutch size, age at first reproduction and longevity.

Main conclusions Lizards with low body temperatures do not seem to have 'slower' life-history attributes than species with high body temperatures. The longer seasons prevalent in warm regions, and physiological processes that operate while lizards are inactive (but warm enough), make environmental temperatures better predictors of lizard life-history variation than body temperatures. This surprisingly greater effect of environmental temperatures on lizard life histories hints that global warming may have a profound influence on lizard ecology and evolution.

\section{Keywords}

Body temperature, diel cycle, environmental temperature, global warming, life history, lizards, thermal biology. 